\title{
CRITICAL BRANCHING RANDOM WALK IN AN IID ENVIRONMENT
}

\author{
JÁNOS ENGLÄNDER AND NÁNDOR SIEBEN
}

\begin{abstract}
Using a high performance computer cluster, we run simulations regarding an open problem about $d$-dimensional critical branching random walks in a random IID environment The environment is given by the rule that at every site independently, with probability $p>0$, there is a cookie, completely suppressing the branching of any particle located there.

The simulations suggest self averaging: the asymptotic survival probability in $n$ steps is the same in the annealed and the quenched case; it is $\frac{2}{q n}$, where $q:=1-p$. This particular asymptotics indicates a non-trivial phenomenon: the tail of the survival probability (both in the annealed and the quenched case) is the same as in the case of non-spatial unit time critical branching, where the branching rule is modified: branching only takes place with probability $q$ for every particle at every iteration.
\end{abstract}

\section{Contents}

1. Introduction

2. Preliminaries

3. Implementation

3.1. Annealed simulation

3.2. Quenched simulation

1

4. Simulation results

4.1. Annealed simulation on $\mathbb{Z}^{2}$

4.2. Annealed simulation on $\mathbb{Z}^{1}$

4.3. Quenched simulation

5. Interpretation of the simulation results

5.1. Main finding

5.2. Interpretation of the fluctuations in the diagrams

6. Beyond the first order asymptotics

6.1. Two dimensions

6.2. One dimension

6.3. Comparison between one and two dimensions

References

\section{INTRODUCTION}

In 3] a spatial branching model has been studied, where the underlying motion is a $d$-dimensional $(d \geq 1)$ Brownian motion, the particles perform dyadic branching, and the branching rate is affected by a random collection of reproduction suppressing sets dubbed mild obstacles. In fact the obstacle configuration was given by the union of balls with fixed radius, where the centers of the balls form a Poisson point process. The radius $r$ plays no role in the results, but the Poisson intensity $\nu>0$ does. The main result of [3] is the quenched Law of Large Numbers for the population for all $d \geq 1$. The environment $\omega$ (with law $P^{\omega}$ )

Date: September 20, 2021.

2000 Mathematics Subject Classification. Primary: 60J80; Secondary: 60K37 .

Key words and phrases. Branching random walk, catalytic branching, mild obstacles, critical branching, random environment, simulation.

The authors thank S. Kuznetsov and R. St. Laurent for helpful conversations. 
has the property that the branching rate is $\beta_{1}$ inside the obstacles and $\beta_{2}$ outside of them, with $0<\beta_{1}<\beta_{2}$. The branching process given the environment $\omega$ was denoted by $Z$. $(\omega)$ and the total population size at $t \geq 0$ was denoted by $\left|Z_{t}(\omega)\right|$. Define the average growth rate by $r_{t}=r_{t}(\omega):=\frac{\log \left|Z_{t}(\omega)\right|}{t}$. In [3] it was shown that for almost every environment,

$$
\lim _{t \rightarrow \infty}(\log t)^{2 / d}\left(r_{t}-\beta_{2}\right)=-c(d, \nu) \quad P^{\omega} \text {-probability, }
$$

where $c(d, \nu)>0$ is an explicit constant. (This is a kind of LLN, because the expectation of the total population size obeys the same asymptotics.)

It has also been shown that the branching Brownian motion with mild obstacles spreads less quickly than ordinary branching Brownian motion, and an upper estimate for its radial speed has been provided.

More general offspring distributions (beyond the dyadic one considered in the main theorems) were also discussed in [3]. In particular, the following question was posed. Consider the model where the offspring distribution is critical. One can easily prove (see Theorem 2.2 below) that, despite of the obstacles, the system still dies out with probability one.

Problem 1.1. What is the rate of decay for the survival probability? Is it still of order $C / n$ as in the obstacle-free (non-spatial) case?

In the present paper we are going to investigate this problem in a discretized setting. More precisely, we consider a modified version of the model, by replacing the Poisson point process with IID probabilities on $\mathbb{Z}^{d}$, as follows. Given an environment, the initial particle, located at the origin, first moves according to a nearest neighbor simple random walk, and immediately afterwards, the following happens to her:

(1) If there is no cookie at the new location, the particle either vanishes or splits into two offspring particles, with equal probabilities.

(2) If there is a cookie at the new location, nothing happens to the particle.

The new generation then follows the same rule in the next unit time interval and produces the third generation, etc.

Let $p \in[0,1]$. In the sequel $\mathbb{P}_{p}$ will denote the law of the cookies and $P^{\omega}$ will denote the law of the BRW given the environment $\omega$. So, if $\mathbf{P}_{p}$ denotes the 'mixed' law in the environment with cookie probability $p$, we have

$$
\mathbf{P}_{p}(\cdot)=\mathbb{E}_{p} P^{\omega}(\cdot) .
$$

Following standard terminology, $P^{\omega}$ and $\mathbf{P}_{p}$ will be called quenched and annealed probabilities, respectively.

We close this section with a remark which is essentially taken from [3] with slight adaptations.

Remark 1.2. An alternative view on our setting is as follows. Let $K$ denote the (random) set of those lattice points where there is a cookie. Then, our model can be viewed as a catalytic BRW as well - the catalytic set is then $K^{c}$ (in the sense that branching is 'made possible' there). Catalytic spatial branching (mostly for superprocesses though) has been the subject of vigorous research in the last twenty years initiated by Dawson, Fleischmann and others — see the survey papers [6] and [2] and references therein. In those models the individual branching rates of particles moving in space depend on the amount of contact between the particle ('reactant') and a certain random medium called the catalyst. The random medium is usually assumed to be a 'thin' random set (that could even be just one point) or another superprocess. Sometimes 'mutually' or even 'cyclically' catalytic branching is considered [2].

Our model is simpler than most catalytic models as our catalytic/blocking areas are fixed, whereas in several catalytic models they are moving. On the other hand, while for catalytic settings studied so far results were mostly only qualitative we are aiming to get quite sharp quantitative results. 
For the discrete setting there are much less results available. One example 1 is [5], where the branching particle system on $\mathbb{Z}^{d}$ is so that its branching is catalyzed by another autonomous particle system on $\mathbb{Z}^{d}$. There are two types of particles, the $A$-particles ('catalyst') and the $B$-particles ('reactant'). They move, branch and interact in the following way. Let $N_{A}(x, s)$ and $N_{B}(x, s)$ denote the number of $A$ - (resp. $B$-)particles at $x \in \mathbb{Z}^{d}$ and at time $s \in[0, \infty)$. (All $N_{A}(x, 0)$ and $N_{B}(x, 0), x \in \mathbb{Z}^{d}$ are independent Poisson variables with mean $\mu_{A}\left(\mu_{B}\right)$.) Every $A$-particle ( $B$-particle) performs independently a continuous-time random walk with jump rate $D_{A}\left(D_{B}\right)$. In addition a $B$-particle dies at rate $\delta$, and, when present at $x$ at time $s$, it splits into two in the next $d s$ time with probability $\beta N_{A}(x, s) d s+o(d s)$. Conditionally on the system of the $A$-particles, the jumps, deaths and splitting of the $B$ particles are independent. For large $\beta$ the existence of a critical $\delta$ is shown separating local extinction regime from local survival regime.

Finally, note that while the continuous equivalent of an IID trap configuration on the lattice is a Poisson trap configuration on $\mathbb{R}^{d}$, there is an important difference between the two. The discrete setting has the advantage that the difference between the sets $K$ and $K^{c}$ is no longer relevant. Indeed, in the discrete case the complement is also IID with a different parameter (self-duality), whereas in the continuous setting this nice duality is lost as the 'Swiss cheese' $K^{c}$ is not the same type of geometric object as $K$; the latter is the case, for example, in [3].

\section{Preliminaries}

In this section we present two simple statements which are relatively easy to verify rigorously. Let $S_{n}$ denote the event of survival for $n \geq 0$. That is, $S_{n}=\left\{Z_{n} \geq 1\right\}$, where $Z_{n}$ is the population size at time $n$.

Theorem 2.1 (Monotonicity). Let $0 \leq p<\widehat{p} \leq 1$ and fix $n \geq 0$. Then

$$
\mathbf{P}_{p}\left(S_{n}\right) \leq \mathbf{P}_{\widehat{p}}\left(S_{n}\right)
$$

Proof. First notice that it suffices to prove the following statement:

Assume that we are given an environment with some 'red' cookies and some additional 'blue' cookies. Then the probability of $S_{n}$ with the additional cookies is larger than or equal to the probability without them.

Indeed, one can argue by coupling as follows. Let $q:=1-p, \delta:=\widehat{p}-p$. First let us consider the cookies that are coming with IID probabilities and parameter $p$. These will be the 'red' cookies. Now with probability $\delta / q$ at each site independently, add a blue cookie. Then the probability for any given site, that there is at least one cookie there is $p+\delta / q-p \delta / q=p+\delta=\widehat{p}$. Now delete those blue cookies where there was a red cookie too. This way, the red cookies plus the additional blue cookies together correspond to parameter $\widehat{p}$.

We are thus going to prove the statement in italics now, using an argument due to S. Kuznetsov. Consider the generating functions of no branching and critical branching: $\varphi_{1}(z)=z$ and $\varphi_{2}(z)=\frac{1}{2}\left(1+z^{2}\right)$, and note that $\varphi_{1} \leq \varphi_{2}$ on $\mathbb{R}$. Fix an environment and define

$$
u(n, x, N):=P_{n, x}\left(S_{N}^{c}\right),
$$

that is, the probability that the population emanating from a single particle which is at time $n$ is located in $x$, becomes extinct at time $N$. Then, if the particle moves to the random location $\xi_{n+1}$, one has

\footnotetext{
${ }^{1}$ A further example of the discrete setting is [1].
} 


$$
\begin{aligned}
u(n, x, N) & =E \sum_{i=0}^{2} p_{i}\left(\xi_{n+1}\right)\left[P_{n+1, \xi_{n+1}}\left(S_{N}^{c}\right)\right]^{i} \\
& =E \sum_{i=0}^{2} p_{i}\left(\xi_{n+1}\right)\left[u\left(n+1, \xi_{n+1}, N\right)\right]^{i}=E \varphi^{\xi_{n+1}}\left[u\left(n+1, \xi_{n+1}, N\right)\right],
\end{aligned}
$$

where $p_{i}\left(\xi_{n+1}\right)$ is the probability 2 of producing $i$ offspring at the location $\xi_{n+1}$, and $\varphi^{\xi_{n+1}}$ is either $\varphi_{1}$ or $\varphi_{2}$.

Now consider two environments: one with only red cookies and another one where there are also some additional blue cookies, and let us denote the corresponding functions by $u_{1}$ and $u_{2}$. We have

and

$$
u_{1}(n, x, N)=E \varphi_{1}^{\xi_{n+1}}\left[u_{1}\left(n+1, \xi_{n+1}, N\right)\right]
$$

$$
u_{2}(n, x, N)=E \varphi_{2}^{\xi_{n+1}}\left[u_{2}\left(n+1, \xi_{n+1}, N\right)\right] .
$$

Clearly $u_{1}(N, x, N)=u_{2}(N, x, N)=0$. Therefore, using that $\varphi_{1} \leq \varphi_{2}$ and by (backward) induction, $u_{2} \geq u_{1}$ for all $n=0,1, \ldots, N-1$. In particular,

$$
u_{1}(0, x, N) \leq u_{2}(0, x, N),
$$

finishing the proof.

We now give a precise statement and a rigorous proof concerning the result about eventual extinction mentioned briefly above.

Theorem 2.2 (Extinction). Let $0 \leq p<1$ and let $A$ denote the event that the population survives forever. Then, for $\mathbb{P}_{p}$-almost every environment, $P^{\omega}(A)=0$.

Proof. Let again $Z_{n}$ denote the total population size at time $n$ for $n \geq 1$. Then $Z$ is a martingale with respect to the canonical filtration $\left\{\mathcal{F}_{n} ; n \geq 1\right\}$. To see this, note that just like in the $p=0$ case, $E\left(Z_{n+1}-Z_{n} \mid \mathcal{F}_{n}\right)=0$, as the particles that do not branch (due to the presence of cookies) do not contribute to the increment. Being a nonnegative martingale, $Z$ converges a.s. to a limit $Z_{\infty}$, and of course $Z_{\infty}$ is nonnegative integer valued. We now show that for $\mathbb{P}_{p}$-almost every environment, $P^{\omega}\left(Z_{\infty}=0\right)=1$. Introduce the events

- $A_{k}:=\left\{Z_{\infty}=k\right\}$ for $k \geq 1$,

- $B$ : branching occurs at infinitely many times $0<\sigma_{1}<\sigma_{2}<\ldots$

Clearly, $A=\cup_{k \geq 1} A_{k}=\left\{Z_{\infty} \geq 1\right\}$. We first show that

$$
\text { for } \mathbb{P}_{p} \text { - a.e. environment, } P^{\omega}\left(B^{c} A\right)=0 \text {. }
$$

Clearly, it is enough to show that $\mathbf{P}_{p}\left(B^{c} A\right)=0$.

Now, $B^{c} A \subset C$, where $C$ denotes the event that there exists a first time $N$ such that for $n \geq N$, there is no branching and particles survive and stay in the region of cookies. On $C$, one can pick randomly a particle starting at $N$, and follow her path; this path visits infinitely many points $P^{\omega}$-a.s. whatever $\omega$ is 3 . Since this path is independent of $\omega$ and $p<1$, the $\mathbb{P}_{p}$-probability that it contains a cookie at each of its sites is zero. Hence $\mathbf{P}_{p}(C)=0$, and (2.1) follows.

On the other hand, for each $k \geq 1$, there is a $p_{k}<1$, such that the probability that the population size remains unchanged (it remains $k$ ) at $\sigma_{m}$ is not more than $p_{k}$ for every $m \geq 1$, uniformly in $\omega$. Thus,

$$
P^{\omega}\left(B A_{k}\right)=P^{\omega}\left(B \cap\left\{Z_{\sigma_{m}}=k \text { for all large enough } m\right\}\right)=0,
$$

whatever $\omega$ is. Using this along with (2.1), we have that for $\mathbb{P}_{p}$-almost every $\omega, P^{\omega}\left(A_{k}\right)=$ $P^{\omega}\left(B^{c} A_{k}\right)+P^{\omega}\left(B A_{k}\right)=0, k \geq 1$, and so $P^{\omega}(A)=0$.

\footnotetext{
${ }^{2}$ So either $p_{1}=1$ or $p_{0}=p_{2}=1 / 2$, according to whether there is no cookie there or there is one.

${ }^{3}$ Because for every $\omega$, it is true $P^{\omega}$-a.s., that every particle that does not branch, has a path that visits infinitely many points
} 


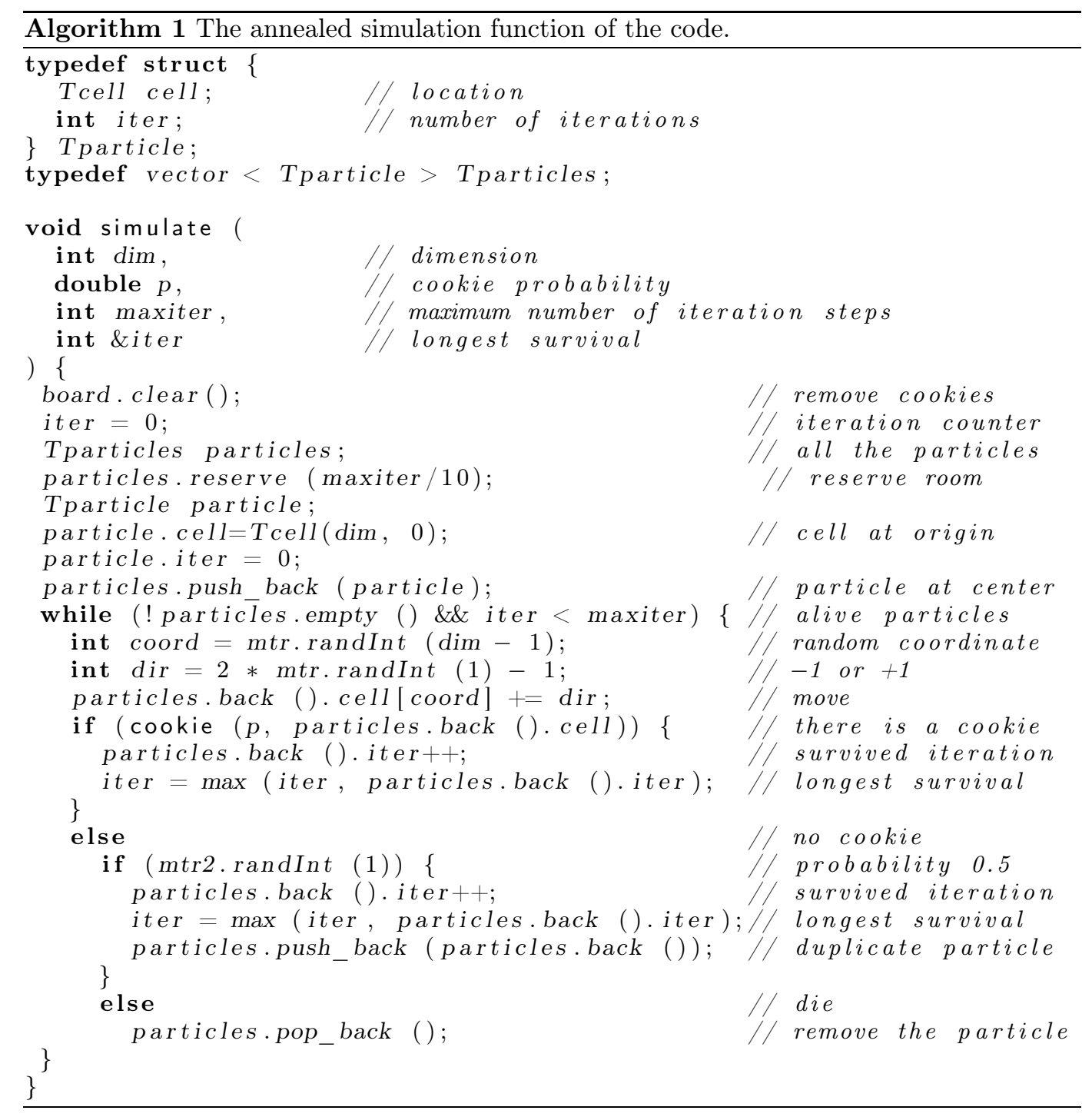

\section{IMPLEMENTATION}

The code for our simulations are written in $\mathrm{C}++$ using the MPIqueue parallel library 8]. We ran the code on 96 cores using a computing cluster containing Quad-Core AMD Opteron(tm) 2350 CPU's. We used an implementation [10] of the Mersenne Twister [7] to generate random numbers. The total running time for the simulations was several months.

3.1. Annealed simulation. Algorithm 1 shows the $\mathrm{C}++$ function that runs a single annealed simulation. We are essentially implementing a 'depth-first search.' Below we give a detailed description of the code.

- line 1: We define a data type to store particles.

- line 2: The location of the particle is stored in the cell field, that is a vector with the appropriate dimensions.

- line 3: The iter field stores the number of iterations survived by the particle.

- line 5: We define a data type to store all the living particles.

- line 7: The simulation function takes three input variables and one output variable.

- line 8: The dimension of the space is the first input.

- line 9: The probability of a cookie at any given location is the second input.

- line 10: The maximum number of allowed iterations is the third input. 
- line 11: The output of the function is the maximum number of iterations any particle survived.

- line 13: We erase all the cookies from the board. Every run of the simulation uses a new cookie placement.

- line 14: The initial value of the output must be zero.

- line 15: We define a variable to store all our alive particles.

- line 16: We reserve some space to store the particles. Making the reserved space too small results in unnecessary reallocation of the variable which degrades performance. On the other hand, reserving too much space can be a problem too since different CPU's compete with each other for RAM.

- lines 18-19: The initial particle starts at the origin before the iterations start.

- line 20: At the beginning we only have the initial particle.

- line 21: We run the simulation while we have alive particles and none of them stayed alive for the maximum allowed number of iterations.

- lines 22-23: We generate a random direction.

- line 24: We move the last of our alive particles in this random direction.

- line 25: We call the cookie function to check if there is a cookie at the new location of the particle. The cookie function checks in the global variable board if any particle already visited this location and as a result we know already whether there is a cookie there. If no particle visited this location before, then the function uses the cookie probability to decide whether to place a cookie there or leave the location empty. This information is then stored for future visitors.

- line 26: If there is a cookie at the new location, then the particle survived one more iteration, so we increment the iter variable.

- line 27: It is possible that this is the longest surviving particle so far, so we update the output variable.

- line 29: If there is no cookie at the new location, then the particle splits or dies.

- line 30: We generate a random number to decide what happens.

- lines 31-32: If the particle splits, then it survives so we update information about the number of iterations.

- line 33: The particle splits, so we place a copy of it into our collection of particles as the last particle.

- lines 35-36: If the particle dies, then we remove it from our collection of particles.

The rest of our code takes care of the parallelization, data collection and the calculation of survival probabilities. The program splits the available nodes into a boss node and several worker nodes. The boss assigns simulation jobs to the workers. The workers call the simulation function several times. The boss node collects the results of these jobs and calculates the survival probabilities using all the available simulation runs. More precisely, $\mathbf{P}_{\mathbf{p}}\left(S_{n}\right)$ is estimated as the number of simulation runs with longest survival value not smaller than $n$ divided by the total number of runs.

3.2. Quenched simulation. The code for quenched simulation is essentially the same with only minor modifications. In this version, line 13 of he simulation function is missing, since we do not want to replace the board at every simulation.

The other change in the simulation function is at line 25. In the annealed case, every worker node has a local version of the board and the cookie function can create the board on the fly. In the quenched case, the worker nodes need to use the same board, so the cookie function cannot generate the board locally. The new version of the cookie function still stores information about the already visited locations. On the other hand, if a location is not visited yet, then the worker node asks the boss node whether this new location has a cookie. The boss node first checks whether the location was visited by any other particle at any other worker node. If the location was visited, then the boss already has a record of this location. Otherwise, the boss node uses the cookie probability to decide whether the location should have a cookie. Essentially, the boss node has the ultimate information 


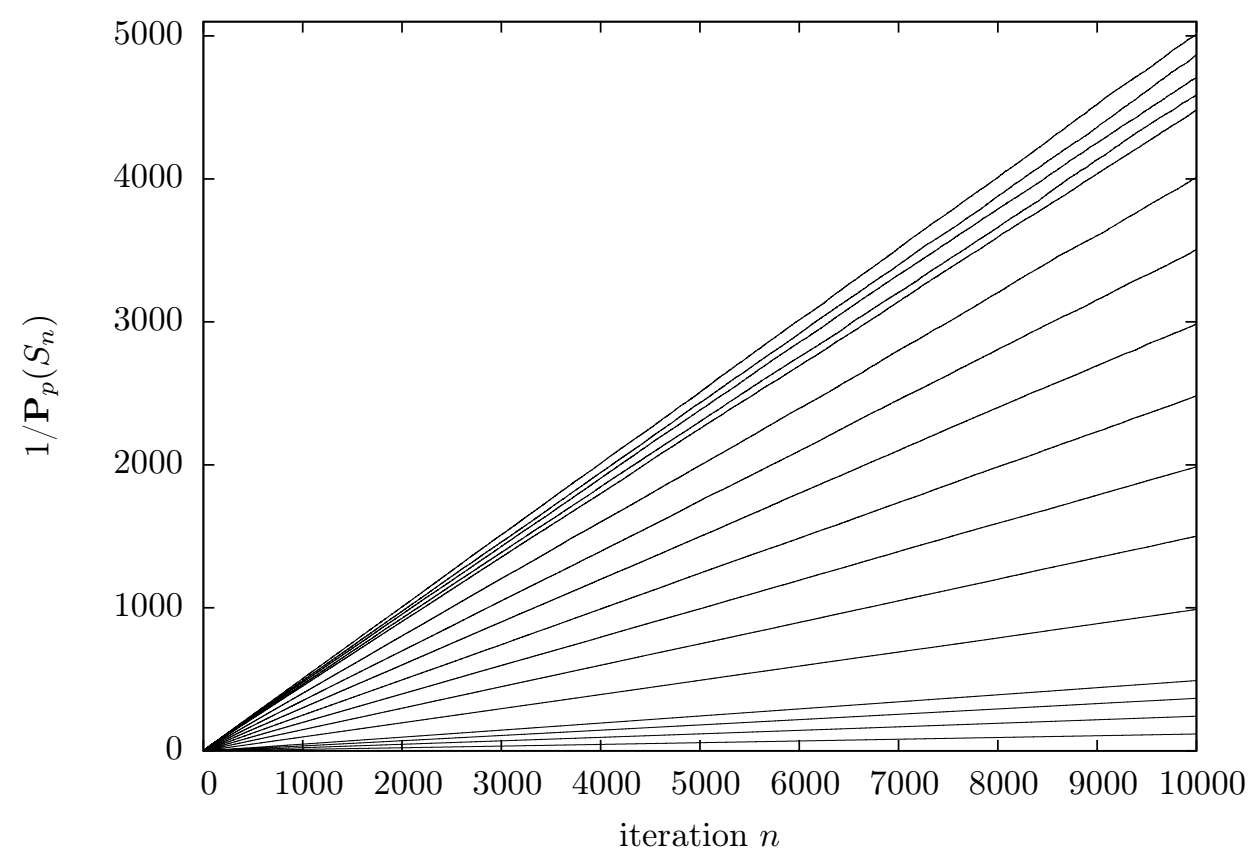

FiguRE 4.1. Results for an annealed 2-dimensional simulation. The graph shows the reciprocal of the survival probability as a function of the number of iterations. Each line represents a different cookie probability. One such line is the result of $10^{8}$ runs of the simulation with a newly generated cookie landscape.

about the board, but the worker nodes keep partial versions of the board and only consult the boss node when it is necessary.

Remark 3.1. In the quenched case, note that if $\widehat{\rho}_{n}$ denotes the relative frequency of survivals (up to $n$ ) after $r$ runs for a fixed environment $\omega$, that is,

$$
\widehat{\rho}_{n}=\widehat{\rho}_{n}^{\omega}:=\frac{\mid \text { survivals } \mid}{r},
$$

then using our method of simulation, the random variables $\widehat{\rho}_{n}$ and $\widehat{\rho}_{m}$ are not independent for $n \neq m$, because the data are coming from the same $r$ runs.

Similarly, in the annealed case, for a fixed environment and a fixed run, the random variables $\mathbf{1}_{S_{n}}$ and $\mathbf{1}_{S_{m}}$ are not independent for $n \neq m$.

\section{Simulation Results}

We ran our annealed simulation on $\mathbb{Z}^{d}$ with $d \in\{1,2,3\}$. The 1-dimensional case turned out to be the most challenging. So we start the description of our results with the 2dimensional case. The 3-dimensional case produced essentially the same output as the 2-dimensional case.

4.1. Annealed simulation on $\mathbb{Z}^{2}$. We executed $10^{8}$ runs allowing a maximum of $n_{\max }=$ 10000 iterations with $p \in\{0,0.025,0.05,0.075,0.1,0.2, \ldots, 0.9,0.925,0.95,0.975\}$. For $p=1$ we used the known survival probability of 1 . Preliminary results made it clear that the simulation is more sensitive for small and large values of $p$. This is why we picked more of these values instead of a uniformly placed set of values. The reciprocal of the calculated survival probabilities are shown in Figure 4.1. The figure suggests that $n \mapsto 1 / \mathbf{P}_{p}\left(S_{n}\right)$ is asymptotically linear. We calculated the slopes for these curves from the values at $4 n_{\max } / 5$ and $n_{\max }$. These slope values are shown in Figure 4.2 


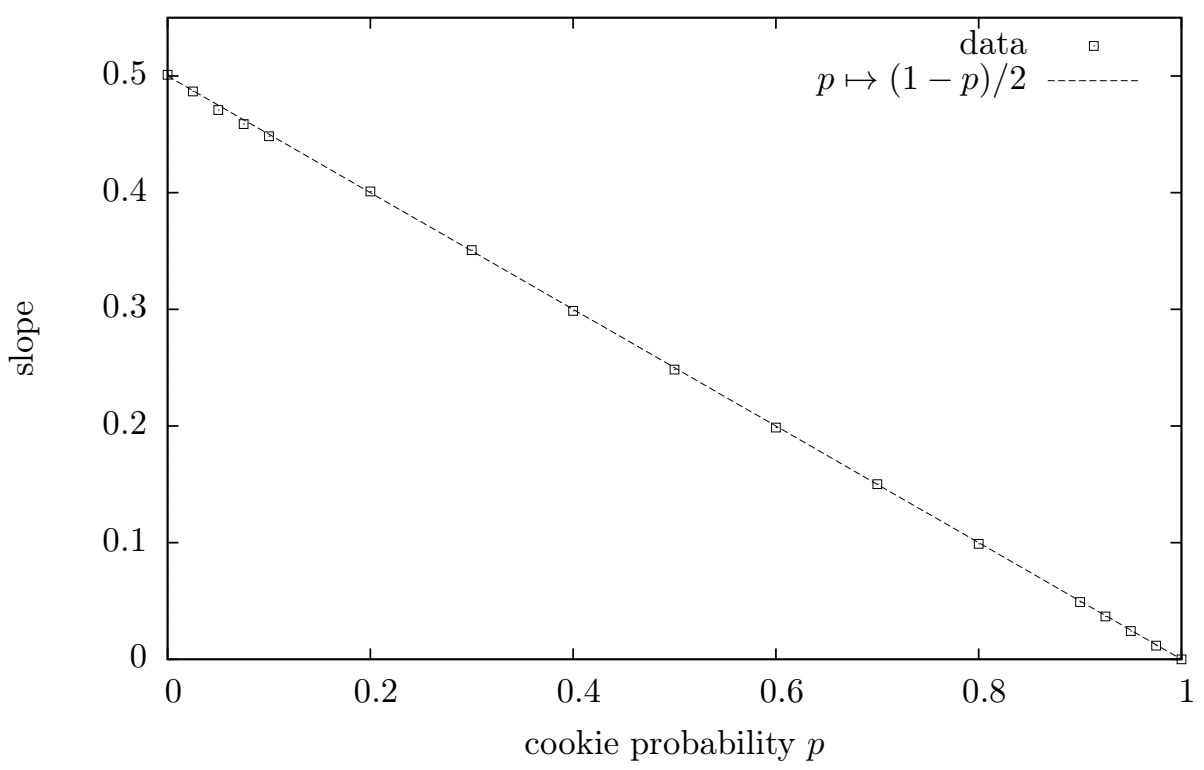

FIgURE 4.2. Results for an annealed 2-dimensional simulation. The graph shows the apparent slopes in Figure 4.1 (i.e. the limits of the functions of Figure 5.1) as a function of the cookie probability together with the graph of $p \mapsto(1-p) / 2$.

\begin{tabular}{|c|c|c|c|c|c|c|}
\hline$p$ & 0 & .5 & .975 & 0 & .5 & .975 \\
\hline$n$ & 1 & 1 & 1 & 2 & 2 & 2 \\
\hline exact & .5 & .75 & .9875 & .375 & .605469 & .975292 \\
\hline simulated & 0.50005 & .74998 & .98749 & .37501 & .605465 & .97528 \\
\hline
\end{tabular}

TABLE 1. Exact and simulated survival probability values $\mathbf{P}_{p}\left(S_{n}\right)$ on $\mathbb{Z}^{2}$. The simulated values are calculated from the data used in Figure 4.1 .

To verify the correctness of our simulation we computed the exact theoretical survival probabilities after the first two iterations. It is easy to see that $\mathbf{P}_{p}\left(S_{1}\right)=1 / 2+p / 2$ and

$$
\mathbf{P}_{p}\left(S_{2}\right)=3 / 8+11 p / 32+3 p^{2} / 16+3 p^{3} / 32 .
$$

Table 1 compares some of the exact and simulated values.

4.2. Annealed simulation on $\mathbb{Z}^{1}$. A 1-dimensional simulation with $10^{8}$ runs and $n_{\max }=$ 10000 produces less satisfactory results as shown in Figure 4.3. The reasons behind this are explained in Subsection 5.2 below, where a discussion is given concerning the fluctuations of the empirical curves in the figures. Essentially, in the annealed case, small values of $\mathbf{P}_{p}\left(S_{n}\right)$ result in large errors (see Subsection 5.2 for more explanation) and therefore we modified the original algorithm by introducing a stopping rule: when the estimated value of $\mathbf{P}_{p}\left(S_{n}\right)$ reaches a certain small threshold value, we stop and do not simulate more iterations. Fortunately, when larger threshold values needed, they are actually large: we obtained slower convergence for large values of $p$, and, clearly, for those values, the probability $\mathbf{P}_{p}\left(S_{n}\right)$ is large. The threshold value was set $1 / 4000$, based on trial and error. This way, we stopped the iteration at $n_{\text {stop }}(p)$; the slopes were then calculated from the values at $4 n_{\text {stop }}(p) / 5$ and $n_{\text {stop }}(p)$. See Figure 4.4.

After adjusting the algorithm by using the above stopping rule, the curve indeed straightened out and the picture became very similar to the 2-dimensional one in Figure 4.2. 


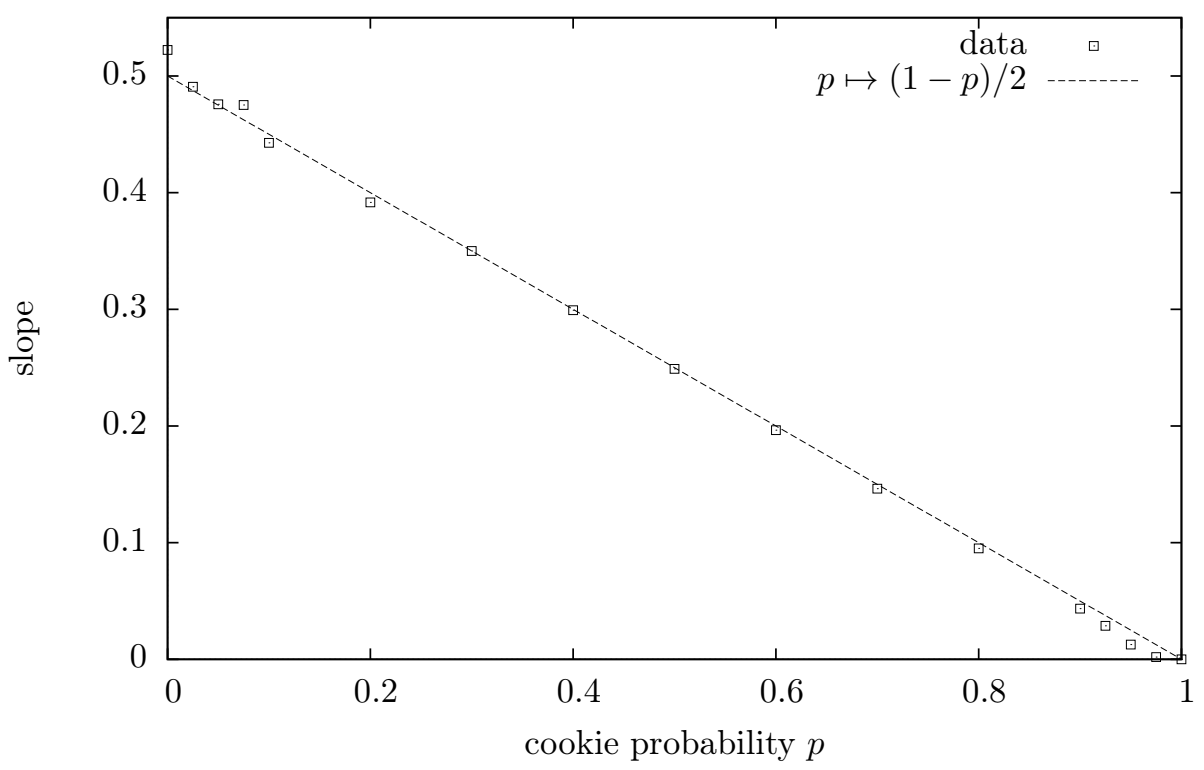

Figure 4.3. Results for an annealed 1-dimensional simulation. Every parameter for this simulation is chosen to be the same as that of Figure 4.2 except the dimension.

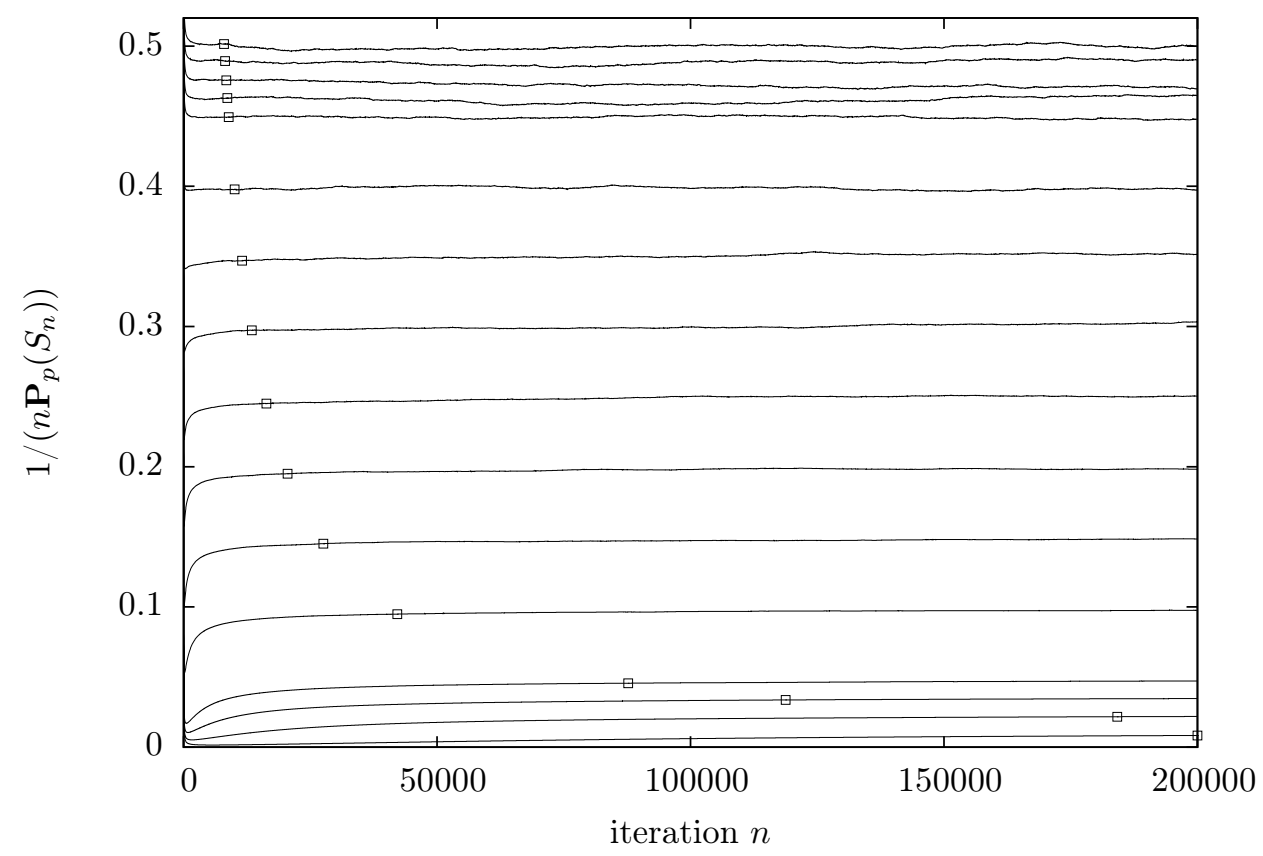

FiguRe 4.4. Annealed 1-dimensional simulation with 959965800 runs. For small values of $p$ (lines at the top), a small iteration number would actually give better results, because otherwise the survival probability $\mathbf{P}_{p}\left(S_{n}\right)$ becomes too small, even with a huge number of runs. On the other hand, for large $p$ values (lines at the bottom) one needs large iteration numbers because the convergence is apparently slow. The squares represent the iteration thresholds after which $\widehat{\rho}_{n}<\frac{1}{4000}$. 


\begin{tabular}{|c|c|c|c|c|c|c|}
\hline$p$ & 0 & .5 & .975 & 0 & .5 & .975 \\
\hline$n$ & 1 & 1 & 1 & 2 & 2 & 2 \\
\hline exact & .5 & .75 & .9875 & .375 & .601563 & .975272 \\
\hline simulated & 0.50002 & .7499992 & .98749 & .37502 & .601569 & .975269 \\
\hline
\end{tabular}

TABLE 2. Exact and simulated survival probability values $\mathbf{P}_{p}\left(S_{n}\right)$ on $\mathbb{Z}^{1}$.

The simulated values are calculated from the data used in Figure 4.4 .

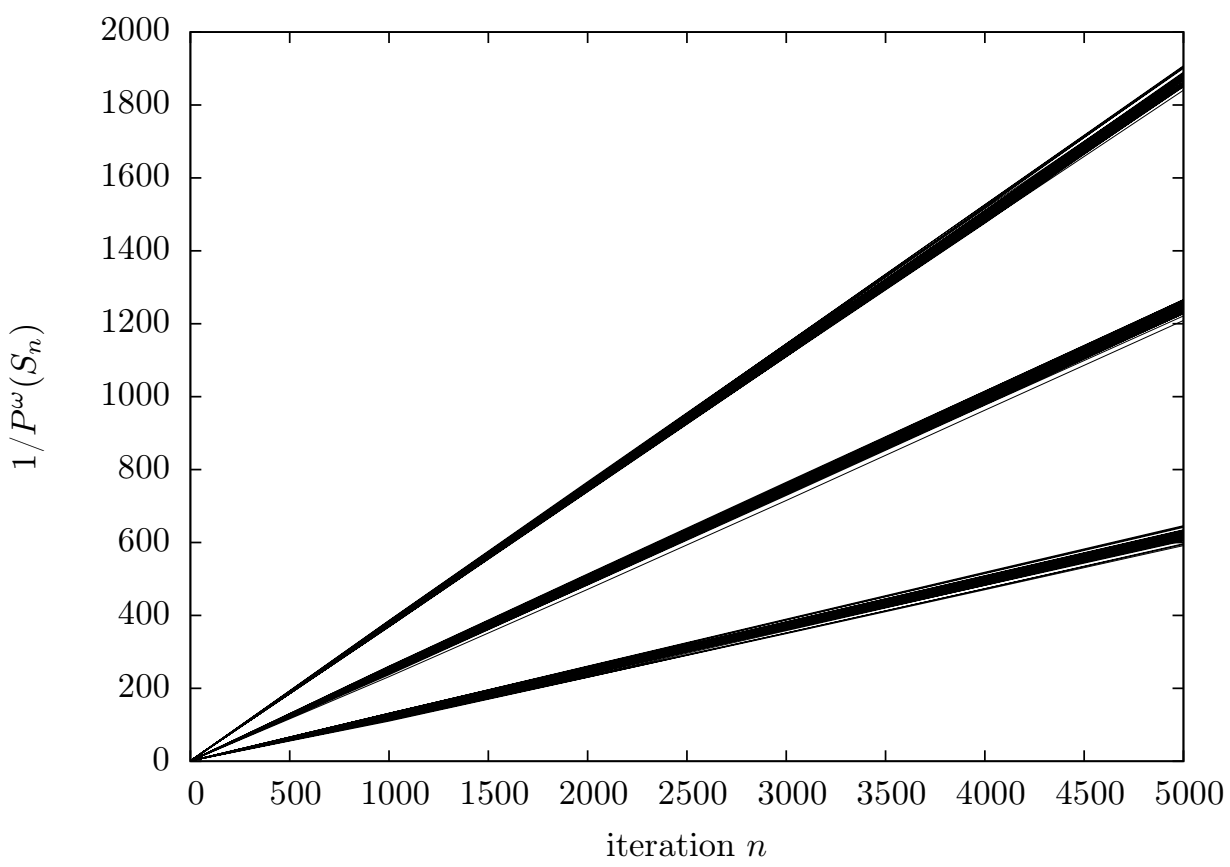

Figure 4.5. Results for a quenched 2-dimensional simulation with $10^{8}$ runs. Each line represents a different cookie landscape. One such line is the result of $10^{8}$ runs of the simulation. The lines are in three groups corresponding to three different cookie probability. Each group has 50 lines. The cookie probabilities from top to bottom are $0.25,0.5$ and 0.75 . The total number of simulations required for this graph is $3 \cdot 50 \cdot 10^{9}$. The total running time was about 29 hours.

To verify the correctness of our simulation we computed the exact theoretical survival probabilities after the first two iterations. It is easy to see that $\mathbf{P}_{p}\left(S_{1}\right)=1 / 2+p / 2$ and

$$
\mathbf{P}_{p}\left(S_{2}\right)=3 / 8+5 p / 16+p^{2} / 4+p^{3} / 16
$$

Table 2 compares some of the exact and simulated values.

4.3. Quenched simulation. From the annealed simulation it has been clear that convergence is much faster in two dimensions than in one dimension. Therefore, in the quenched case we chose to present our results in the $d=2$ case. In fact, we got qualitatively similar results for $d=1$.

In Figure 4.5 we see three bundles corresponding to three values of $p$. Those bundles are very thin, so essentially the same thing happens for every realization, and the slopes of the lines are roughly $3 / 8,1 / 4$ and $1 / 8$ from top to bottom, corresponding to $p=0.25, p=0.5$ and $p=0.75$, respectively. That is, for each one of these values of $p$, the slope is the same as in the annealed case. 
Although Figure 4.5 is about the $d=2$ case, we have a similar simulation result for $d=1$; in fact we conjecture that this qualitative behavior (that is, the coincidence of the first order asymptotics of the quenched and annealed survival probability) will hold for all $d \geq 1$.

\section{INTERPRETATION OF THE SIMULATION RESUlTS}

5.1. Main finding. Recall first the classic result due to Kolmogorov [4, Formula 10.8], that for critical unit time branching with generating function $\varphi$, as $n \rightarrow \infty$,

$$
P(\text { survival up to } n) \sim \frac{2}{n \varphi^{\prime \prime}(1)} .
$$

As a particular case, let us consider now a non-spatial toy model as follows. Suppose that branching occurs with probability $q \in(0,1)$, and then it is critical binary, that is, consider the generating function

$$
\varphi(z)=(1-q) z+\frac{1}{2} q\left(1+z^{2}\right) .
$$

It then follows that, as $n \rightarrow \infty$,

$$
P(\text { survival up to } n) \sim \frac{2}{q n} \text {. }
$$

Turning back to our spatial model, the simulations suggest (Figures 4.1 and 4.5) the self averaging property of the model: as explained in the previous section, the asymptotics for the annealed and the quenched case are the same. In fact, this asymptotics is the same as the one in (5.1), where $p=1-q$ is the probability that a site has a cookie. In other words, despite our model being spatial, in an asymptotic sense, the parameter $q$ simply plays the role of the branching probability of the above non-spatial toy model. To put it yet another way, $q$ only introduces a 'time-change.'

The intuitive picture behind this asymptotics is that there is nothing either the environment or the BRW could do to increase the chance of survival, at least as far as the leading order term is concerned (as opposed to well known models, for example when a single Brownian motion is placed into random medium [9]). Hence, given any environment the particles move freely and experience branching at $q$ proportion of the time elapsed (quenched case), and the asymptotics agrees with the one obtained in the non-spatial setting as in (5.1). Furthermore, creating a 'good environment' (annealed case) and staying in the part of the lattice with cookies for very long would be 'too expensive.'

Note that whenever the total population size reduces to one, the probability of that particle staying in the region of cookies is known to be much less than $\mathcal{O}(1 / n)$ (hard obstacle problem for random walk). So the optimal strategy for this particle to survive is obviously not to try to stay completely in that region and thus avoid branching. Rather, survival will mostly be possible because of the potentially large family tree stemming from that particle. In fact, the formula $\mathbf{P}_{p}\left(S_{n}\right) \sim \frac{2}{q n}$, together with the martingale property of $\left|Z_{n}\right|$, implies that $\mathbf{E}_{p}\left(\left|Z_{n}\right| \mid S_{n}\right) \sim \frac{q}{2} \cdot n$.

Notice that the straight lines on Figures 4.2 and 4.3 start at the value $1 / 2$, that is, as $p \downarrow 0$, one gets the well known non-spatial asymptotics $2 / n$, which is a particular case of Kolmogorov's result above. We conclude that there is apparently no discontinuity at $p=0$ (no cookies) for the quantity $\lim _{n \rightarrow \infty} n P$ (survival up to $n$ ).

5.2. Interpretation of the fluctuations in the diagrams. Since we estimated the reciprocal of the survival probabilities and not the probabilities themselves both in the annealed and the quenched case (Figures 4.1 and 4.5), one cannot expect good approximation results when those probabilities are small. Indeed, in the annealed case, if $\rho_{n}:=\mathbf{P}_{p}\left(S_{n}\right)$ (with $p$ being fixed) and $\widehat{\rho}_{n}$ denotes the relative frequency obtained from simulations, then the Law of Large Numbers (LLN) only says, that if the number of runs is large, then the difference $\left|\rho_{n}-\widehat{\rho}_{n}\right|$ is small. However, looking at the difference of the reciprocals

$$
\left|\frac{1}{\rho_{n}}-\frac{1}{\widehat{\rho}_{n}}\right|=\frac{\left|\rho_{n}-\widehat{\rho}_{n}\right|}{\rho_{n} \widehat{\rho}_{n}},
$$




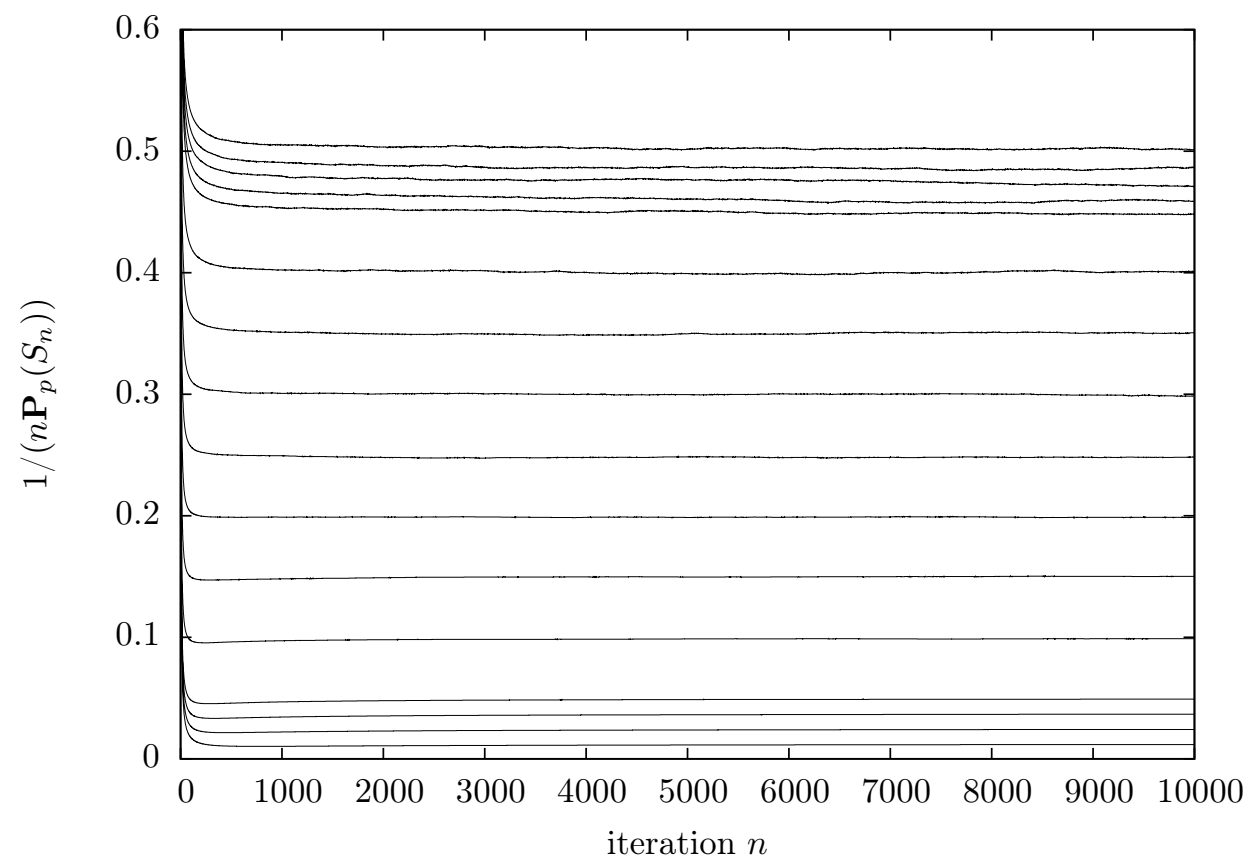

FiguRE 5.1. Results for an annealed 2-dimensional simulation with $10^{8}$ runs. The graph shows the reciprocal of the survival probability divided by the number of iterations as a function of the number of iterations. The data used to create the graph is the same as that of Figure 4.1

it is clear that a small $\rho_{n}$ value magnifies the error; in fact the effect is squared as $\widehat{\rho}_{n}$ is close to $\rho_{n}$, exactly because of the LLN. This effect is the reason of the 'zigzagging' of the line on Figure 4.3 for small $p$ values. In fact, small $p$ values result in small $\rho_{n}$ values in light of Theorem 2.1 and the continuity property mentioned at the end of the previous subsection. Clearly, there is a competition between $\rho_{n}$ being small (as a result of $p$ being small and $n$ being large) on the one hand, and the large number of runs on the other. The first makes the denominator small in $\frac{\left|\rho_{n}-\widehat{\rho}_{n}\right|}{\rho_{n} \widehat{\rho}_{n}}$, while the second makes the numerator small according to LLN.

Looking at Figure 4.3, one notices another peculiarity in the 1-dimensional setting. For large values of $p$, the empirical curve is slightly under the straight line. The explanation for the relatively poor fit is simply that the iteration number is not large enough for the asymptotics to 'kick in.'

These arguments are bolstered by the experimental findings that increasing the number of runs helps for small $p$ values, whereas increasing the number of iterations helps for large ones. For example, in Figure 4.4 we increased the maximal iteration number $n_{\max }$ to 200000 and plotted $n \mapsto\left(n \mathbf{P}_{p}\left(S_{n}\right)\right)^{-1}$. One can see that for small $p$ values it is beneficial to stop the iterations earlier, but for large values large iteration numbers give better results.

We do not have an explanation, however, for the deviation downward from the straight line (for large $p$ values) in Figure 4.3. We suggest, as an open problem, to find at least a heuristic explanation for this phenomenon.

Interestingly, for higher dimensions there is apparently a perfect fit for large values of $p$, indicating that for higher dimensions the asymptotics is much quicker then for $d=1$. Figure 5.1 checks the assumption (for $d=2$, annealed) that the reciprocal of the survival probability is $\frac{q n}{2}+o(n)$ as $n \rightarrow \infty$, by dividing the reciprocal of the survival probability by $n$. The graphs convincingly show the existence of a limit, depending on $p$. 


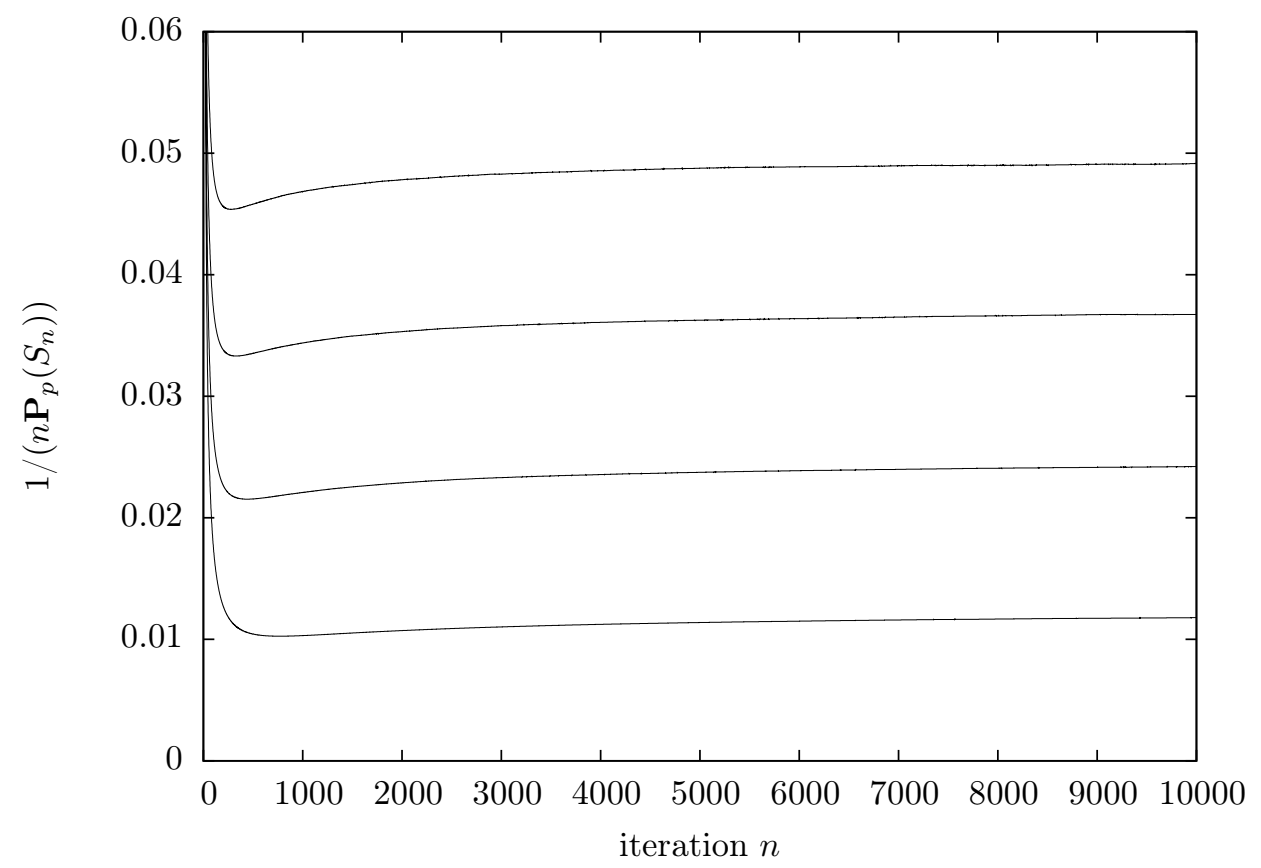

Figure 5.2. Zooming in at the bottom part of Figure 5.1 (i.e. large $p$ values): the convergence is apparently from below.

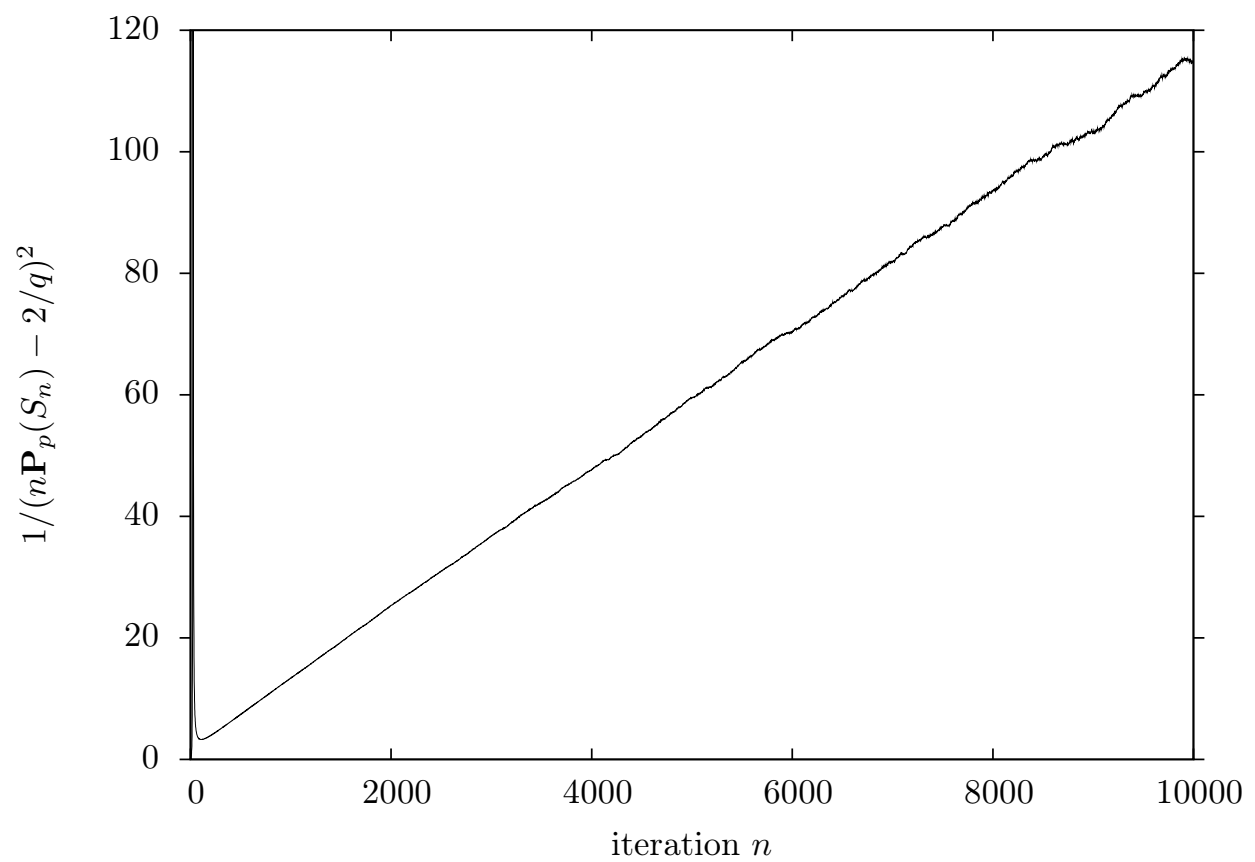

Figure 6.1. Annealed 1-dimensional simulation with 7,259,965,800 runs and $p=0.5$. 


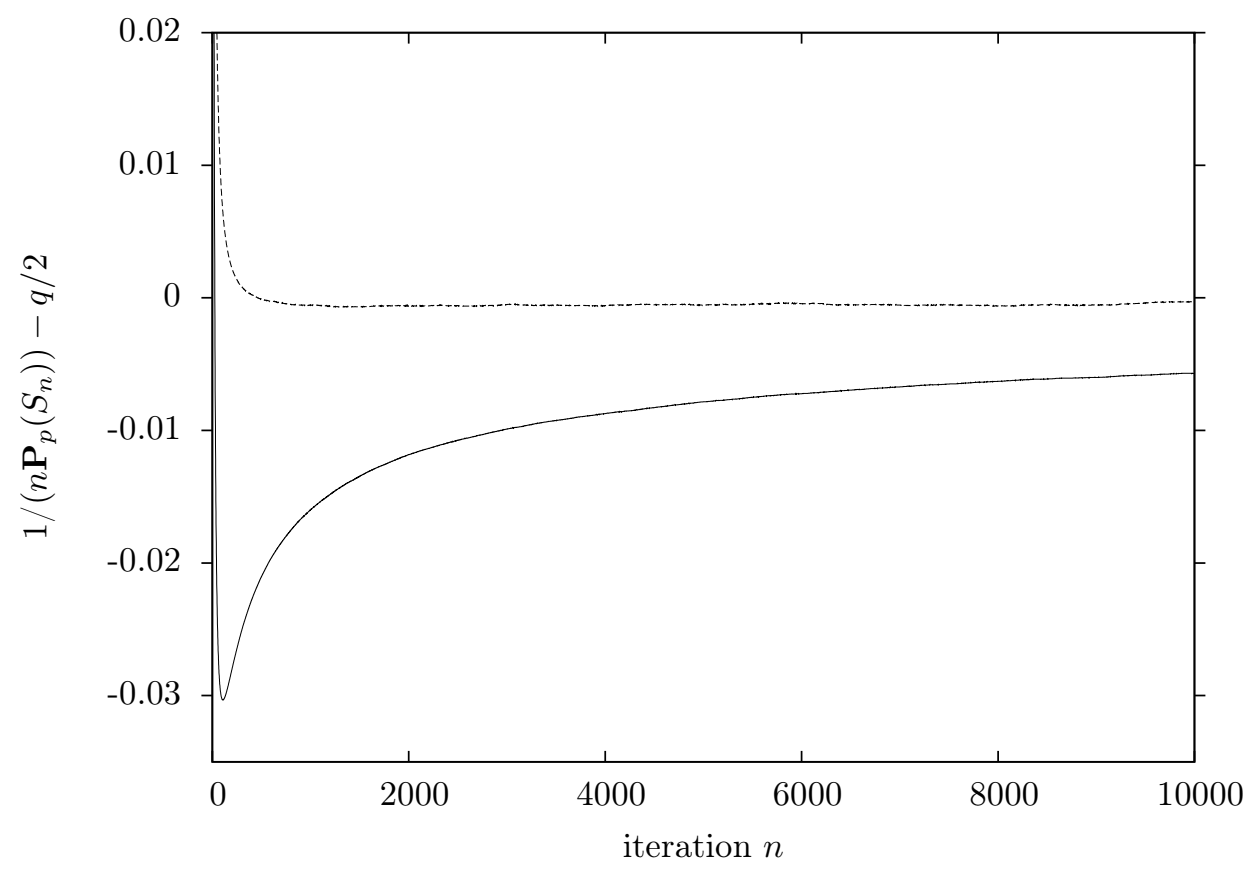

Figure 6.2. Annealed simulation with $p=0.5$. The solid curve shows the 1-dimensional result, the dashed curve shows the 2-dimensional result.

\section{BEYOND THE FIRST ORDER ASYMPTOTICS}

In this section we will attempt to draw conclusions about more delicate phenomena beyond the first order asymptotics, and those conclusions will necessarily be less reliable than the ones in the previous sections.

6.1. Two dimensions. Consider again Figure 5.1 Zooming in gives Figure 5.2. Looking at Figure 4.4, Figure 5.1 and Figure 5.2, for small $p$ values (top lines) the convergence seems to be from above, and for large $p$ values it seems to be from below.

6.2. One dimension. For $d=1$ we obtained figures somewhat similar to the 2-dimensional ones, which we summarize below without actually providing them. Simulation seems to suggest that for not too small values of $p$, the convergence is also from below; this is in line with the fact that, as we have already discussed, on Figure 4.3 the 1-dimensional empirical curve is below the straight line for large $p$ values. For very small $p$ 's, the direction of the convergence is not clear from the pictures. Although the convergence is apparently quicker, the effects are 'blurred' due to the magnification of error explained earlier.

The following conjecture is based on Figure 6.1

Conjecture 6.1 (Second order asymptotics). The 1-dimensional annealed survival probability obeys the following second order asymptotics:

$$
\mathbf{P}_{p}\left(S_{n}\right)=\frac{2}{n q}+f(n)
$$

where $\lim _{n \rightarrow \infty} f(n) \cdot n^{3 / 2}=C>0$, and $C$ may depend on $p$.

6.3. Comparison between one and two dimensions. The annealed convergence to the limit $2 / q$ seems to be quite different for $d=1$ and $d=2$. Figure 6.2 shows this difference, and in particular, it illustrates that in 1-dimension, the convergence is slower and it is apparently from below for $p=0.5$. 


\section{REFERENCES}

1. Sergio Albeverio and Leonid V. Bogachev, Branching random walk in a catalytic medium. I. Basic equations, Positivity 4 (2000), no. 1, 41-100.

2. D. A. Dawson and K. Fleischmann, Catalytic and mutually catalytic super-Brownian motions, Seminar on Stochastic Analysis, Random Fields and Applications, III (Ascona, 1999), Progr. Probab., vol. 52, Birkhäuser, Basel, 2002, pp. 89-110.

3. János Engländer, Quenched law of large numbers for branching Brownian motion in a random medium, Ann. Inst. Henri Poincaré Probab. Stat. 44 (2008), no. 3, 490-518.

4. Theodore E. Harris, The theory of branching processes, Dover Phoenix Editions, Dover Publications Inc., Mineola, NY, 2002, Corrected reprint of the 1963 original [Springer, Berlin; (29 \#664)].

5. Harry Kesten and Vladas Sidoravicius, Branching random walk with catalysts, Electron. J. Probab. 8 (2003), no. 5, 51 pp. (electronic).

6. Achim Klenke, A review on spatial catalytic branching, Stochastic models (Ottawa, ON, 1998), CMS Conf. Proc., vol. 26, Amer. Math. Soc., Providence, RI, 2000, pp. 245-263.

7. Makoto Matsumoto and Takuji Nishimura, Mersenne twister: a 623-dimensionally equidistributed uniform pseudo-random number generator, ACM Trans. Model. Comput. Simul., no. 1, 3-30.

8. John M. Neuberger, Nándor Sieben, and James W. Swift, MPIqueue: A simple library implementing parallel job queues in $C++$, (preprint).

9. Alain-Sol Sznitman, Brownian motion, obstacles and random media, Springer Monographs in Mathematics, Springer-Verlag, Berlin, 1998.

10. Richard Wagner, http://www-personal.umich.edu/ wagnerr/MersenneTwister.html.

Department of Mathematics, University of Colorado, Boulder, CO-80309-0395, and Department of Mathematics and Statistics, Northern Arizona University, Flagstaff, AZ-86011.

E-mail address: Janos.Englander@Colorado.edu, nandor.sieben@nau.edu

URL: http://euclid.colorado.edu/ englandj/MyBoulderPage.html, http://jan.ucc.nau.edu/ ns46 